\title{
HEREDITARY HOMOTOPY EQUIVALENCES
}

\author{
ALLAN J. SIERADSKI
}

\begin{abstract}
This paper introduces the notion of hereditary homotopy equivalence which provides a homotopy-theoretic reformulation of the existence of a CohenLyndon basis for a group presentation.
\end{abstract}

1. Introduction. Let $(X: R)$ be a group presentation satisfying this Relator Condition: No relator $r \in R$ is a proper power, nor a conjugate of another relator $r^{\prime} \in R$ or its inverse. Let $F(X)$ denote the free group on the set $X$ of generators and let $N(R)$ be the normal closure in $F(X)$ of the set $R$ of relators. We consider the following combinatorial-group-theoretic conditions for the presentation $(X: R)$ :

(1) $N(R)$ has a free (Cohen-Lyndon) basis $\mathscr{B}=\cup_{r \in R}\left\{u r u^{-1}: u \in U(r)\right\}$, where for each $r \in R, U(r)$ is a full left transversal for $N(R)$ in $F(X)$.

(2) Every identity sequence for $(X: R)$ reduces to the empty sequence by Peiffer exchanges and Peiffer deletions.

(3) Every identity for $(X: R)$ is a Peiffer identity.

In [1], where the Relator Condition is not imposed, Chiswell, Collins, and Huebschmann refer to a group presentation satisfying a version of (1) as CohenLyndon aspherical (CLA), to one satisfying (2) as diagrammatically aspherical (DA), and to one satisfying (3) as aspherical (A). They observe the implications CLA $\Rightarrow$ $\mathrm{DA} \Rightarrow \mathrm{A}$ (the latter in the presence of the Relator Condition).

Statements (1)-(3) have the following homotopy-theoretic reformulation in terms of the cellular model $K=K(X: R)$ (see $\$ 2)$ of the group presentation $(X: R)$ and its universal covering complex $\tilde{K}$ :

$\left(1^{*}\right) \tilde{K}$ has the hereditary homotopy type of a sum of discs with the minimal cell structure.

(2*) Every spherical map into $K$ admits a geometrically split null homotopy.

$\left(3^{*}\right) K$ is aspherical.

The equivalence (3) $\equiv\left(3^{*}\right)$ is classical and follows from Reidemeister's description [4] of $\pi_{2}(K)$ as the group of identities modulo the group of Peiffer identities (see [5] for a current proof). The equivalence $(2) \equiv\left(2^{*}\right)$ is established in [5], where it is employed to show that the reverse of the implication $\mathrm{DA} \Rightarrow \mathrm{A}$ is not valid (a fact also established by an example in [1]). Finally, the equivalence $(1) \equiv\left(1^{*}\right)$ and the

Received by the editors December 8, 1981 and, in revised form, March 15, 1982.

1980 Mathematics Subject Classification. Primary 57M20, 55P15, 20 F05.

Key words and phrases. Cohen-Lyndon basis, hereditary homotopy equivalences, aspherical complexes. group presentations. 
notion of a hereditary homotopy equivalence are new and form the subject matter for this paper. The homotopy-theoretic viewpoint of $\left(1^{*}\right)$ and $\left(2^{*}\right)$ may help resolve the status of the reverse of the implication CLA $\Rightarrow \mathrm{DA}$, a point left open by [1].

2. Hereditary homotopy equivalences. A hereditary homotopy equivalence $F: M \rightarrow K$ between 2-dimensional $\mathrm{CW}$ complexes is a homotopy equivalence of their skeleton pairs $F:\left(M^{2}, M^{1}\right) \rightarrow\left(K^{2}, K^{1}\right)$ which restricts to a homotopy equivalence $F \mid N$ : $\left(N, M^{1}\right) \rightarrow\left(F(N), K^{1}\right)$ for each subcomplex $M^{1} \subset N \subset M^{2}$. We may assume that the image $F(N)$ is a subcomplex of $K$, since $F \mid N$ homotopes off any cells of $K$ which it fails to cover. It is clear the the composition of hereditary homotopy equivalences is again hereditary. So the associated relation on the class of 2-dimensional $\mathrm{CW}$ complexes, defined by $M \stackrel{H}{\equiv} K$ if and only if there exists a hereditary homotopy equivalence $F: M \rightarrow K$, is transitive, as well as reflexive. The symmetry of this relation is not so obvious since we have not demanded that a homotopy inverse to $F$ be hereditary also. Nevertheless, this symmetry is available as a consequence of the proposition of this section. So the relation $\equiv$ partitions the class of 2-dimensional $\mathrm{CW}$ complexes into equivalence classes, which we call hereditary homotopy types.

For ease in presenting examples, we adopt this notation: The cellular model $K(X: R)$ of a group presentation $(X: R)$ is a 2-complex $K=c^{0} \cup c_{x}^{1} \cup c_{r}^{2}(x \in$ $X, r \in R$ ) whose cells are oriented by characteristic maps $\theta_{x}: B^{1} \rightarrow K$ and $\varphi_{r}$ : $B^{2} \rightarrow K$ such that there is an identification $\pi_{1}\left(K^{1}\right) \equiv F(X)$, under which the cellular path $\theta_{x}$ represents $x \in F(X)$ and the attaching map $\dot{\varphi}_{r}=\varphi_{r} \mid S^{1}$ represents $r \in R \subseteq$ $F(X)$.

EXAMPLES. Let $M$ and $K$ be the contractible models of the presentations ( $a, b$ : $a, a b)$ and $(x, y: x, y)$ of the trivial group. The cellular map $F: M \rightarrow K$, for which $F \theta_{a}=\theta_{x}, F \theta_{b}=\theta_{y}, F \varphi_{a}=\varphi_{x}$, and $F \varphi_{a b}=\varphi_{x} \cdot \varphi_{y}$, carries the open cells $c_{a}^{1}, c_{b}^{1}$, and $c_{a}^{2}$ homeomorphically onto the open cells $c_{x}^{1}, c_{y}^{1}$, and $c_{x}^{2}$, while it pinches the open cells $c_{a h}^{2}$ into the union $c_{x}^{2} \cup c^{0} \cup c_{y}^{2}$. This map is a homotopy equivalence of pairs $F$ : $\left(M^{2}, M^{1}\right) \rightarrow\left(K^{2}, K^{1}\right)$, but it is not a hereditary homotopy equivalence since it does not restrict to a homotopy equivalence on the subcomplex $N=M^{1} \cup c_{a b}^{2}$.

On the other hand, the cellular map $G: M \rightarrow K$, given by $G \boldsymbol{\theta}_{a}=\boldsymbol{\theta}_{x}, G \boldsymbol{\theta}_{h}=\boldsymbol{\theta}_{y} \cdot \boldsymbol{\theta}_{x}^{-1}$, $G \varphi_{a}=\varphi_{x}$, and $G \varphi_{a b}=\theta_{x} * \varphi_{y}\left(\right.$ where $*$ gives the action of $\pi_{1}\left(K^{\prime}\right)$ on $\left.\pi_{2}\left(K^{2}, K^{1}\right)\right)$, is a hereditary homotopy equivalence. This map carries the open cells $c_{a}^{1}$ and $c_{a}^{2}$ homeomorphically onto the open cells $c_{x}^{1}$ and $c_{x}^{2}$; it wraps the open cell $c_{b}^{1}$ around both loops of the bouquet $c_{x}^{1} \cup c^{0} \cup c_{v}^{1}$, while it collapses the open cell $c_{a b}^{2}$ onto the union $c_{x}^{1} \cup c^{0} \cup c_{y}^{2}$. It is seen to restrict to a homotopy equivalence on both subcomplexes of $M$.

Let $M^{1} \cup_{\dot{\varphi}} c_{\alpha}^{2}$ denote the 2-complex obtained from a 1-dimensional CW complex $M^{\prime}$ by adjoining 2-cells $\left\{c_{\alpha}^{2}\right\}$ via the family $\dot{\varphi}$ of attaching maps $\left\{\dot{\varphi}_{\alpha}: S^{1} \rightarrow M^{1}\right\}$. There are these two basic construction techniques for hereditary homotopy equivalences:

(4) Any homotopy equivalence $F: M^{1} \rightarrow K^{1}$ of 1-dimensional $\mathrm{CW}$ complexes extends to a hereditary homotopy equivalence $F: M^{1} \cup_{\dot{\varphi}} c_{\alpha}^{2} \rightarrow K^{1} \cup_{F \dot{\varphi}} c_{\alpha}^{2}$. 
(5) The identity map 1: $K^{1} \rightarrow K^{1}$ on any 1-dimensional $\mathrm{CW}$ complex extends to a hereditary homotopy equivalence $G: K^{1} \cup_{\psi} c_{\alpha}^{2} \rightarrow K^{1} \cup_{\dot{\varphi}} c_{\alpha}^{2}$ if the attaching maps $\dot{\varphi}_{\alpha}$ and $\dot{\psi}_{\alpha}$ are freely homotopic in $K^{1}$ for all $\alpha$.

The proofs [2, p. 40] of the standard (nonhereditary) versions of these claims are valid here, and they produce homotopy inverses for $F$ and $G$ which are also hereditary.

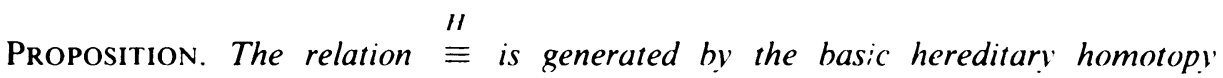
equivalences (4) and (5).

Proof. Let $F: M \rightarrow K$ be a given hereditary homotopy equivalence. For each single 2-cell subcomplex $N=M^{1} \cup_{\dot{\varphi}} c^{2}$ of $M$, the image $F(N)$ is a single 2-cell subcomplex $L=K^{1} \cup_{\psi} c^{2}$ of $K$. since the homotopy equivalence $F \mid N:\left(N, M^{1}\right) \rightarrow$ $\left(L, K^{1}\right)$ gives a homotopy equivalence $S^{2} \equiv N / M^{1} \rightarrow L / K^{1}$ between a single 2-sphere and a bouquet of 2-spheres in 1:1 correspondence with the 2-cells of $L$. Then the commutative ladder of isomorphisms, induced by the homotopy equivalence $F \mid N$, between the exact homotopy sequences for the pairs $\left(N, M^{\prime}\right)$ and $\left(L, K^{\prime}\right)$ shows that the maps $F \dot{\varphi}$ and $\dot{\psi}$ are freely homotopic to loops at the base point of $K^{1}$ whose homotopy classes have the same normal closure in the free group $\pi_{1}\left(K^{1}\right)$. Thus, by the Freiheitzsatz [3, II 6] one of these homotopy classes is a conjugate in $\pi_{1}\left(K^{1}\right)$ of the other or its inverse, or equivalently, the maps $F \dot{\varphi}$ and $\dot{\psi} \cdot$ ' are freely homotopic in $K^{1}$. This condition for each of the paired 2-cells in $M$ and $K$ implies that there is a hereditary homotopy equivalence $M \rightarrow K$ obtained as a composite of a basic one of type (4) followed by one of type (5).

By this proposition, and the fact that the basic hereditary homotopy equivalences (4) and (5) have homotopy inverses which are also hereditary, it follows that the relation $\stackrel{H}{\equiv}$ is symmetric, and hence is an equivalence relation, as noted at the start of this section.

3. Cohen-Lyndon basis and special contractions. Let $p: \tilde{K} \rightarrow K$ be the covering projection for the universal covering space $\tilde{K}$ of the cellular model $K=K(X: R)$ of a group presentation $(X: R)$. The covering space $\tilde{K}$ inherits from $K=c^{0} \cup c_{x}^{1} \cup c_{r}^{2}$ $(x \in X, r \in R)$ a cell structure $\tilde{K}=\tilde{c}_{g}^{0} \cup \tilde{c}_{(g, x)}^{1} \cup \tilde{c}_{(g, r)}^{2}(x \in X, r \in R, g \in G=$ $F(X) / N(R))$. There are characteristic maps $\theta_{(g, x)}: B^{1} \rightarrow \tilde{K}^{1}$ and $\varphi_{(g, r)}: B^{2} \rightarrow \tilde{K}$ such that $\boldsymbol{\theta}_{(g, x)}$ is a path from $\tilde{c}_{g}^{0}$ to $\tilde{c}_{g x}^{0}$ for which $p \theta_{(g, x)}=\theta_{x}$, and the attaching map $\dot{\varphi}_{(g, r)}=\varphi_{(g, r)} \mid S^{1}$ is a loop at $\tilde{c}_{g}^{0}$ for which $p \dot{\varphi}_{(g, r)}=\dot{\varphi}_{r}$.

THEOREM. The following are equivalent for a group presentation $(X: R)$ satisfying the Relator Condition:

(1) $N(R)$ has a (Cohen-Lyndon) basis $\mathscr{B}=\cup_{r \in R}\left\{\right.$ uru $\left.{ }^{-1}: u \in U(r)\right\}$, where for each $r \in R, U(r)$ is a full left transversal for $N(R)$ in $F(X)$.

$\left(1^{*}\right) \tilde{K}$ has the hereditary homotopy type of a sum $\vee B^{2}$ of discs with the minimal $C W$ decomposition.

Proof. (1) $\Rightarrow\left(1^{*}\right)$. For each pair $(g, r) \in G \times R$, we may express $g \in G$ as a coset $u N(R)$ for a unique transversal element $u=u_{(g, r)} \in U(r) \subseteq F(X)$. Then we 
may represent $u \in F(X) \equiv \pi_{1}\left(K^{1}\right)$ by a loop $\alpha_{u}$ in $K^{1}$ at $c^{0}$, and lift this loop through the covering projection $p$ to a path $\tilde{\alpha}_{u}$ in $\tilde{K}^{1}$ from $\tilde{c}_{1}^{0}$ to $\tilde{c}_{g}^{0}$. Then the attaching loop $\dot{\varphi}_{(g, r)}$ at $\tilde{c}_{g}^{0}$ is freely homotopic in $\tilde{K}^{1}$ to the loop $\dot{\psi}_{(g, r)}=\tilde{\alpha}_{u} \cdot \dot{\varphi}_{(g . r)} \cdot \tilde{\alpha}_{u}^{-1}$ at $\tilde{c}_{1}^{0}$. So by (5) there is a basic hereditary homotopy equivalence

$$
G: \tilde{K}^{1} \cup_{\psi} c_{(g, r)}^{2} \rightarrow \tilde{K}^{1} \cup_{\dot{\varphi}} c_{(g, r)}^{2}=\tilde{K}
$$

Under the identifications

$$
\pi_{1}\left(\tilde{K}^{\prime}\right) \stackrel{p_{*}}{\equiv} \operatorname{Ker}\left(\pi_{1}\left(K^{\prime}\right) \rightarrow \pi_{1}(K)\right) \equiv N(R)
$$

we have corresponding elements $\left[\dot{\psi}_{(g, r)}\right] \leftrightarrow\left[\alpha_{u} \cdot \dot{\varphi}_{r} \cdot \alpha_{u}^{-1}\right] \leftrightarrow u r u^{-1}$ for all $g=u N(R)$, $u \in U(r)$, and $r \in R$. Since $\mathscr{B}=\bigcup_{r \in R}\left\{u r u^{-1}: r \in U(r)\right\}$ is a free basis for $\pi_{1}\left(\tilde{K}^{1}\right)$ $\equiv N(R)$, the map $F: M^{1}=\bigvee S_{(g . r)}^{1} \rightarrow \tilde{K}^{1}$, given by $F \mid S_{(g . r)}^{1}=\dot{\psi}_{(g . r)}$, is a homotopy equivalence, and hence by (4) $F$ extends to a hereditary homotopy equivalence $F$ : $\vee B_{(g, r)}^{2} \rightarrow \tilde{K}^{1} \cup_{\psi} c_{(g, r)}^{2}$.

The composite $G F: \vee B_{(g, r)}^{2} \rightarrow \tilde{K}$ is the desired hereditary homotopy equivalence.

For the converse $\left(1^{*}\right) \Rightarrow(1)$, we invoke the proof of the proposition of $\$ 2$ to replace a given hereditary homotopy equivalence by one $F: \vee B_{(g . r)}^{2} \rightarrow \tilde{K}$ for which the loop $F \mid S_{(g . r)}^{1}$ at $\tilde{c}_{1}^{0}$ is freely homotopic to the loop $\dot{\varphi}_{(g . r)}$ at $\tilde{c}_{g}^{0}$. It follows that $F \mid S_{(g . r)}^{1}$ is based homotopic to a path product $\tilde{\alpha}_{(g, r)} \cdot \dot{\varphi}_{(g, r)} \cdot \tilde{\alpha}_{(g, r)}^{-1}$, for some path $\tilde{\alpha}_{(g . r)}$ in $\tilde{K}^{\prime}$ from $\tilde{c}_{1}^{0}$ to $\tilde{c}_{g}^{0}$. Then for fixed $r \in R$, the loops $\alpha_{(g . r)}=p \tilde{\alpha}_{(g . r)}$ in $K^{1}$ at $c^{0}$ represent elements $u=u_{(g . r)} \in F(X) \equiv \pi_{1}\left(K^{\prime}\right)$ of a full left transversal $U(r)$ for $N(R)$ in $F(X)$. The isomorphisms

$$
\pi_{1}\left(\vee S_{(g, r)}^{1}\right) \stackrel{F_{*}}{\cong} \pi_{1}\left(\tilde{K}^{1}\right) \stackrel{p_{\text {\# }}}{\equiv} N(R)
$$

show that $N(R)$ has a Cohen-Lyndon basis $\because h=\cup_{r \in R}\left\{u r u^{-1}: u \in U(r)\right\}$ associated with the free basis $\left\{\left[F \mid S_{(g, r)}^{1}\right]\right\}$ of $\pi_{1}\left(\tilde{K}^{1}\right)$. This completes the proof.

In view of the equivalence $(3) \equiv\left(3^{*}\right)$, an aspherical presentation $(X: R)$ is one for which the covering complex $\tilde{K}$ for the cellular model $K=K(X: R)$ is contractible. By the equivalence $(1) \equiv\left(1^{*}\right)$, a CLA presentation $(X: R)$ is one for which $\tilde{K}$ contracts in a very special manner. The examples in [1] or [5] of aspherical presentations which are not DA, hence not CLA, show that the type of contractibility described by $\left(1^{*}\right)$ is restrictive. This is even more transparent in the case of an aspherical presentation for the trivial group. We have this specialization of the preceding theorem.

COROLlARY. The following are equivalent for a group presentation $(X: R)$ with a contractible model $K=K(X: R)$ :

(1) $F(X)$ has a free basis $\mathscr{B}=\left\{u_{r} r u_{r}^{-1}: r \in R\right\}$ for some elements $u_{r} \in F(X)$, $r \in R$.

$\left(1^{*}\right) K$ has the hereditary homotopy type of a sum $\vee B^{2}$ of discs with the minimal $C W$ decomposition.

For example, the model $K$ of the group presentation $\left(x, y: x, y x y^{-1} x y x^{-1}\right)$, which is known to be not DA [5, p. 134], hence not CLA, cannot have the hereditary 
homotopy type of a sum $B^{2} \vee B^{2}$ of discs. This is established directly by the Corollary, since there do not exist elements $u, v \in F(x, y)$ such that $u x u^{-1}$ and $v\left(y x y^{-1} x y x^{-1}\right) v^{-1}$ is a free basis for $F(x, y)$.

\section{REFERENCES}

I. I. M. Chiswell, D. J. Collins and J. Huebschmann, Aspherical group presentations, Math. Z. 178 (1981), 1-36.

2. P. J. Hilton, Homotopy theory and duality, Gordon and Breach, New York, 1965.

3. R. C. Lyndon and P. E. Schupp, Combinatorial group theory, Ergebnisse der Math. und ihrer Grenzgebiete, Bd. 89, Springer, Berlin and New York, 1977.

4. K. Reidemeister, Über Identitäten von Relationen, Abh. Math. Sem. Univ. Hamburg 16 (1949), $114-118$.

5. A. J. Sieradski, Framed links for Peiffer identities, Math. Z. 175 (1980), 125-137.

Department of Mathematics, University of Oregon, Eugene, Oregon 97403 